Chris Freiling, Math Dept., California State University, San Bernardino, CA 92407, e-mail: cfreilin@@wiley.csusb.edu

Robert W. Vallin, Math Dept., Slippery Rock University, Slippery Rock, PA 16057, e-mail: rwv@@sruvm.sru.edu

\title{
SIMULTANEOUS RECOVERY OF BAIRE ONE FUNCTIONS
}

\begin{abstract}
Given any countable collection of Baire one functions there is a single trajectory from which each of the functions is first return recoverable.
\end{abstract}

O'Malley [5] introduced the notion of first return paths to study differentiation properties of real functions. This launched a flurry of explorations. In one of the most intriguing of these, Darji, Evans, and O'Malley [2] (see also [1]) propose a creative and exciting new way to characterize Baire-one functions. They show that the function values can be described by a limiting process which is closely associated with continuity. This process is called "first return recovery". We consider here the possibility of simultaneously recovering countably many such functions, and by doing so, provide an alternate viewpoint for the proof of the Darji, Evans, O'Malley Theorem.

Any countable dense sequence of real numbers $T=\left\{t_{1}, t_{2}, \ldots\right\}$ will be called a "trajectory". For each real number $x$ we say that " $t_{i}$ is in the path to $x$ ", denoted $t_{i} \in T(x)$, if and only if none of the elements $\left\{t_{j}\right\}_{j<i}$ are between $t_{i}$ and $x$. Let us say that $T$ "approximates" a set $A$ (just for the purposes of this paper; it is not our intention to introduce more terminology to this already crowded field) if and only if $(\forall x \in A) x \in T$ or $T(x) \backslash A$ is finite. Our main result is that any countable collection of closed sets can be approximated by a single trajectory.

\footnotetext{
Key Words: first return recovery, Baire class one

Mathematical Reviews subject classification: Primary: 26A03. Secondary: 26A21,

Received by the editors November 3, 1994

* Research supported by NSF.
} 26A15 
This theorem will easily generalize to other spaces. But since there may be several natural interpretations of "between" in the definition of $T(x)$, we concentrate here only on the space of real numbers.

Theorem Given any countable collection of closed sets, $F_{1}, F_{2}, \ldots$, there is a trajectory, $T$, which approximates each $F_{i}$.

Proof. Write the rational numbers as $\left\{q_{i}\right\}_{i=1}^{\infty}$. We will create our trajectory, $T$, in stages, one stage for each rational number. At the $i^{\text {th }}$ stage, $q_{i}$ will be put into our trajectory (if it is not in there already) along with a finite collection of other numbers, $T_{i}$, which we now describe.

With each real number $x$ we associate the string $\sigma(x) \in\{0,1\}^{i}$ in the following manner:

1 in the $j^{\text {th }}$ place means $x \in F_{j}$

0 in the $j^{\text {th }}$ place means $x \notin F_{j}$.

We order these strings lexicographically; that is, $\sigma(x)<\sigma(y)$ if and only if in the first entry where they differ, $\sigma(x)$ has a zero while $\sigma(y)$ has a one. Note that although the rule for $\sigma$ is the same at each stage, the length of the associated strings will be growing. This of course will not reverse any of the inequalities; if $\sigma(x)<\sigma(y)$ at a particular stage, this inequality will continue to hold at future stages. This fact will be useful to us later. For each real number $x$, we denote by $A(x)$ the collection of points $y$ such that $\sigma(y)>\sigma(x)$ and for some finite intersection of the sets $F_{1}, \ldots, F_{i}$ (call this intersection $F)$, either $y$ is the maximum of $F \cap(-\infty, x]$ or the minimum of $F \cap[x, \infty)$. The set $A(x)$ has at most $2^{i+1}$ elements. For a finite set $S$, we let $A(S)$ denote the finite set $\cup_{s \in S} A(s)$. Notice that at future stages $j>i$, the operation $A$ only increases; this fact will also be used later. Finally, we let $T_{i}$ denote the set, starting with $q_{i}$, which is closed under the operation $A$. That is, $T_{i}=\left\{q_{i}\right\} \cup A\left(q_{i}\right) \cup A\left(A\left(q_{i}\right)\right) \cup \ldots$, a process which must eventually stop because there are only finitely many strings at stage $i$.

Before we move on to $q_{i+1}$ we must first describe which order the elements of $T_{i}$ are placed in our trajectory. First, ignore any element which is already in the trajectory. Then, put in the others according to the lexicographic order induced by $\sigma$, so that those elements with the largest string are put in first. Among elements which are tied, that is, which have the same string, we don't care about the order. We now continue to the next stage, adjoining the elements of $T_{i+1}$, and so on, building up our trajectory $T$.

Let us now suppose that some $F_{i}$ is not approximated by $T$. Then choose the least such $i$ and let $x \in F_{i}$ with $x \notin T$ and $T(x) \backslash F_{i}$ infinite. With only finitely many exceptions, each $y \in T(x)$ has the following properties:

1) The number $y$ is put into the trajectory at some stage $j>i$. 
2) The strings $\sigma(x)$ and $\sigma(y)$ agree on the first $i-1$ places (since the sets $F_{1}, \ldots, F_{i-1}$ are both closed and approximated by $T$.)

Fix such a $y$ and, using that $T(x) \backslash F_{i}$ is infinite, assume also that $y \notin F_{i}$. Let $F$ be the intersection of the sets $F_{1}, \ldots, F_{i}$ which contain $x$, and choose $z$ to be the closest element of $F$ to $y$, on the side of $y$ containing $x$. Then using the mapping $\sigma$ defined above at stage $i$, we have $\sigma(z)>\sigma(y)$ since $z \in F_{i}, y \notin F_{i}$, and by property 2 ). Therefore, $z \in A(y)$ (also defined above at stage i). Using property 1) the relations $\sigma(z)>\sigma(y)$ and $z \in A(y)$ also hold at stage $j$. Then $z$ must also be in the trajectory and in fact, is put in there before $y$. Since $x \notin T, z$ can't be $x$, so $z$ is strictly between $x$ and $y$. But then $y \notin T(x)$ after all.

Definition A function $f$ is "recovered" by a trajectory $T$ if and only if $(\forall x \notin$ $T) \lim _{y \in T(x)} f(y)=f(x)$.

Note This is an abuse of the original terminology and is slightly stronger than the "recovery" used in [1]. For a single Baire one function, proof of this stronger type of recovery follows from the techniques in [1] and is explicitly stated and proved in [3]. This stronger type of recovery generalizes a theorem of Gleyzal [5] which says that a Baire one function is the limit of an interval function. Indeed, suppose $f(x)$ is recovered using a trajectory $T$. For each closed interval $I$, let $H(I)=f(x)$ where $x$ is the first element of $T$ in $I$. The fact that for all $x, \lim _{|I| \rightarrow 0, x \in I} H(I)=f(x)$ follows immediately from the definition of "recovered" given above. Finally, observe that to recover the characteristic function of a singleton $\{x\}$ requires that $x$ be in the trajectory. For this reason, simultaneous recovery of uncountably many such functions is impossible.

Corollary Given a countable collection of Baire-one functions there is a single trajectory which recovers each of them.

Proof. Take the inverse image of each open rational interval under each function, obtaining a countable collection of $F_{\sigma}$ sets. Express each of these sets as a countable union of closed sets and consider the entire collection closed sets thus obtained. Then find a trajectory $T$, which simultaneously approximates each set in the collection. Now fix a particular function $f$ and a particular $x \notin T$. For any rational open interval $I$ containing $f(x)$, all but finitely many elements of $T(x)$ are mapped into $I$. Therefore, $\lim _{y \in T(x)} f(y)=f(x)$.

\section{References}

[1] U. B. Darji and M. J. Evans, Recovering Baire 1 functions, Mathematika, 42 (1995), 43-48. 
[2] U. B. Darji, M. J. Evans and R. J. O'Malley, A first return characterization of Baire one functions, Real Anal. Exch., 19 (1993-94), 510-515.

[3] M. J. Evans and R. J. O'Malley, Fine tuning the recoverability of Baire one functions, Real Analysis Exch., 21 (1995-96), 165-174.

[4] A. Gleyzal, Interval-functions, Duke Math. J., 8 (1941), 223-230.

[5] R. J. O'Malley, First return path derivatives, Proc. Amer. Math. Soc., 116 (1992), 73-77. 